\title{
Implementierung einer geriatrischen frührehabilitativen Komplexbehandlung (GFK) in der akutstationären Pneumologie
}

\author{
Implementation of an Early Geriatric Rehabilitation in Acute Inpatient \\ Pneumology
}

Autoren

Institute
W. Ammenwerth ${ }^{1}$, M. Nosul ${ }^{2}$, M. Berliner ${ }^{2}$, Ch. Duve' ${ }^{1}$, A. Guttenberger-Nowicki ${ }^{3}$, N. Schönfeld ${ }^{1}$, T. Blum ${ }^{1}$, T. T. Bauer ${ }^{1}$

Klinik für Pneumologie, Lungenklinik Heckeshorn, HELIOS Klinikum Emil von Behring, Berlin

2 Zentrum für Geriatrie und Physikalische Medizin, HELIOS Klinikum Berlin-Buch, Berlin

Sozialdienst, HELIOS Klinikum Emil von Behring, Berlin eingereicht 10.11 .2011 akzeptiert nach Revision 9. 2. 2012

\section{Bibliografie}

Dol http://dx.doi.org/ 10.1055/s-0031-1291874 Pneumologie 2012; 66: 235-239

(c) Georg Thieme Verlag KG Stuttgart · New York ISSN 0934-8387

\section{Korrespondenzadresse} Dr. Wilhelm Ammenwerth HELIOS Klinikum Emil von Behring Klinik für Pneumologie Lungenklinik Heckeshorn Walterhöferstraße 11 14165 Berlin wim.ammenwerth@helioskliniken.de

\section{Zusammenfassung \\ $\nabla$}

Hintergrund: Um den sich rasch entwickelnden Funktionsverlusten bei insbesondere älteren Patienten mit akut exazerbierter COPD frühzeitig entgegenzuwirken, wurde das Konzept „GFK in der akutstationären Pneumologie“ entwickelt. Wesentlicher Aspekt des Projekts ist die zielgruppenspezifische Ausrichtung unter Nutzung multiprofessioneller Fachkompetenz und Standards. Methode: In dieser 1-jährigen Projektstudie wurden 58 akut exazerbierte COPD-Patienten in höherem Lebensalter (Mittel 74,8 $\pm 6,8$ Jahre) mit geriatrietypischer Multimorbidität und akut- sowie rehabilitationsmedizinischem Behandlungspotenzial eingeschlossen. Mittels standardisierten Assessments wurde das Ergebnis der GFK durch ein multiprofessionelles Reha-Team im prospektiven Ansatz überprüft.

Ergebnisse: Die GFK begann im Median am 3.Tag (Spannweite 1.-22.Tag) und dauerte im Median 16 Tage (Spannweite 9-29). Es konnte insbesondere in den Bereichen Mobilität (Timed-upand-go, Median 19 (Spannweite 10-150) vs. 15 (Spannweite 7-120) Sekunden, $p<0,0001$ ), Selbsthilfefähigkeit (Barthel-Index, Median 73 (Spannweite 5-95) vs. 95 (Spannweite 45-100) Punkte, $p<0,0001$ ) und soziale Versorgung eine signifikante Verbesserung erreicht werden.

Schlussfolgerung: Eine GFK bei akut exazerbierten COPD-Patienten ist hervorragend in die pneumologische akutstationäre Behandlung integrierbar.

\section{Einleitung}

\section{$\nabla$}

Für die Effizienz einer ambulanten und stationären pneumologischen Rehabilitation, insbesondere bei der COPD, gibt es mittlerweile hinreichende Evidenz [ 1 - 10]. In einer 2009 von Puhan und seinen Mitarbeitern veröffentlichten Metaanalyse zeigten sich eindeutige Effekte sowohl bezüglich

\section{Abstract \\ $\nabla$}

Background: In order to counter the rapidly developing loss of function especially in elderly patients with acute exacerbation of chronic obstructive pulmonary disease (AE-COPD) the concept "early geriatric rehabilitation in acute inpatient pneumology" was developed. An essential aspect of the project was a targeted approach making use of multi-professional expertise and standards.

Methods: This 1-year feasibility study included a total of 58 patients with AE-COPD in advanced age (mean: $74.8 \pm 6.8$ years) with typical geriatric multimorbidity and necessity for acute medical as well as rehabilitation treatment. The results of the early geriatric rehabilitation by a multi-professional rehabilitation team were analyzed in a prospective study approach using standardized assessments.

Results: The early geriatric rehabilitation started on median day 3 (range: 1 st -22nd) and lasted in median 16 days (range: 9 -29). It achieved a significant improvement, particularly in mobility [timed up-and-go, median 19 (range: $10-150$ ) vs. 15 (range: $7-120$ ) seconds, $p<0.0001$ ], selfhelp ability [Barthel index, median 73 (range: 5 -95) vs. 95 (range: $45-100$ ) points, $p<0.0001$ ] and social care.

Conclusions: Early geriatric rehabilitation in a cohort of AE-COPD patients is feasible and can be integrated in an acute inpatient pulmonary care system.

der stationären Wiederaufnahmerate (number needed to treat $[\mathrm{NNT}]=3$ ) als auch bezüglich der nachfolgenden Mortalität $(\mathrm{NNT}=6)$ [3]. Alle Behandlungsmaßnahmen in diesen Studien wurden in dafür spezialisierten ambulanten oder stationären Rehabilitationseinrichtungen durchgeführt, bei akut exazerbierten COPD-Patienten setzen rehabilitative Maßnahmen in der Regel erst 
nach der Akutbehandlung ein [11-14]. In den meisten Studien fehlt die Berücksichtigung der Komorbidität, obwohl sie in hohem Maße bei COPD-Patienten vorhanden ist [15].

Für den geriatrischen Patienten, der aufgrund der demografischen Entwicklung auch in der Pneumologie eine stark wachsende Rolle spielt, existiert derzeit kein fachlich verbundenes geriatrisch-pneumologisches Konzept für die stationäre Akutbehandlung.

Am Beispiel des Schlaganfalls, als einem etablierten geriatrischen Krankheitsbild, konnte gezeigt werden, dass ein akuter Funktionsverlust umso stärker rückgängig gemacht werden konnte, je früher Rehabilitationsmaßnahmen einsetzten [16,17]. Der frühestmögliche Beginn der Rehabilitation ist bereits der Zeitpunkt der stationären Aufnahme im Akutkrankenhaus. Unsere Hypothese ist, dass nur so das große Rehabilitationspotenzial der Frühphase optimal genutzt werden kann.

Daher haben wir im Sinne einer Machbarkeitsstudie die Integration einer geriatrischen frührehabilitativen Komplexbehandlung (GFK) in die stationäre akut-pneumologische Versorgung geprüft.

\section{Methode}

$\nabla$

Das multiprofessionelle Behandlungsteam der GFK in der pneumologischen Akutklinik setzte sich aus qualifizierten Ärzten, Therapeuten und Fachkräften unter Leitung eines ärztlichen Koordinators wie folgt zusammen:

- geriatrisch qualifizierter Arzt (Facharzt für Geriatrie aus dem Zentrum für Geriatrie und Physikalische Medizin des HELIOS Klinikum Berlin-Buch mit vollzeitiger Anstellung im HELIOS Klinikum von Behring, Trägerklinik der Lungenklinik Heckeshorn) mit fachärztlicher Behandlungsleitung

- Facharzt für Pneumologie aus dem pneumologischen Akutkrankenhaus (Lungenklinik Heckeshorn)

- Physiotherapeut/Krankengymnast

- Atmungstherapeut

- Ergotherapeut

- Pflegefachkraft mit spezieller Schulung/Qualifikation

- Sozialarbeiter

- Ernährungsberater/Diätassistent

- Logopäde

- Seelsorger/Theologe

Zur Erbringung der GFK in der pneumologischen Akutklinik wurde lediglich eine Vollzeitstelle für einen Facharzt für Geriatrie eingerichtet. Alle weiteren infrastrukturellen Voraussetzungen waren im Klinikum bereits gegeben. Die Abrechnung der GFK erfolgte im Rahmen des DRG-Systems (DRG E42Z, OPS-Kode 8-550.1-3).

In die GFK eingeschlossen wurden im Zeitraum von 09/2010 bis 08/2011 Patienten mit folgenden Merkmalen:

- akut-exazerbierte chronisch-obstruktive Lungenerkrankung (COPD)

- geriatrietypische Multimorbidität

- höheres Lebensalter (Alter > 70 Jahre), wobei die geriatrietypische Multimorbidität im Sinne des biologischen Alters Vorrang vor dem kalendarischen Alter hatte

- manifeste Fähigkeitsstörungen

- Rehabilitationsfähigkeit

- kurativer, akutmedizinischer und rehabilitationsmedizinischer Behandlungsbedarf
Jeder neu stationär aufgenommene Patient mit COPD wurde auf die o.g. Kriterien hin für die Eignung der GFK evaluiert. Der Patient wurde über die Behandlungsmaßnahmen im Rahmen der GFK aufgeklärt. Die Zustimmung des Patienten war Voraussetzung für den Beginn der Rehabilitationsmaßnahme.

$\mathrm{Zu}$ den Eingangsuntersuchungen gehörte die leitliniengerechte pneumologische Diagnostik der COPD inklusive Lungenfunktionsanalyse mit spirometrischer Messung der 1-Sekunden-Kapazität (syn. Forciertes Einsekundenvolumen, FEV1), der inspiratorischen Vitalkapazität (VC) und die Bestimmung des Verhältnisses FEV1/VC für die Beurteilung der Atemwegsobstruktion $[18,19]$. Als Referenzwerte für die Spirometrie bei Erwachsenen dienten die Empfehlungen der Europäischen Gemeinschaft für Kohle und Stahl [20]. Körpergröße und -gewicht wurden für die Berechnung des Body Mass Index (BMI; Körpergewicht in Kilogramm geteilt durch Quadrat der Körpergröße in Metern) gemessen. Außerdem wurde ein standardisiertes geriatrisches Aufnahmeassessment in den Bereichen Selbsthilfefähigkeit (Barthel-Index=BI), Mobilität (Timed-up-and-go=TUG), Kognition (MiniMental-Status =MMST), Emotion (Geriatrische Depressionsskala =GDS-4) und soziale Versorgung (Soziales Assessment durch den Sozialdienst) gemäß ICD-10-GM U50-U52 [21] durchgeführt. Vor Entlassung des Patienten aus der pneumologischen Akutklinik wurde das Ergebnis der GFK mittels standardisiertem Entlassungsassessment in den Bereichen Selbsthilfefähigkeit (BI) und Mobilität (TUG) überprüft. Obligat wurde ein Entlassungsmanagement durchgeführt, dazu gehörte die Vorbereitung der ambulanten medizinischen, pflegerischen, physiotherapeutischen und sozialen Versorgung.

Im Rahmen der täglichen aktivierend-therapeutischen Pflege wurden Mobilisierung, Körperpflege, An- und Auskleiden, Essen und Trinken, Aktivierungstherapie, Pneumonie-, Sturz- und Thromboseprophylaxe durchgeführt. Die Patienten erhielten mehr als 10 Therapieeinheiten pro Woche jeweils von mindestens 30 Minuten aus den Therapiebereichen Physiotherapie, Ergotherapie und physikalische Therapie. Zudem wurde jeder Patient von einem Ernährungsberater, einem Atmungstherapeuten und einem Seelsorger konsultiert. Es fand eine multiprofessionelle, wöchentliche Teambesprechung mit wochenbezogener Dokumentation bisheriger Behandlungsergebnisse und weiterer Behandlungsziele statt.

Somit wurden bei allen Patienten die Vorgaben des OPS-Kodes 8-550 erfüllt (OPS 2011, Operationen- und Prozedurenschlüssel Internationale Klassifikation der Prozeduren in der Medizin).

Die Auswertung aller Daten erfolgte mit dem Statistikprogramm IBM $^{\circledR}$ SPSS $^{\circledR}$ Statistics 19 für Windows ${ }^{\circledR}$. Bei der deskriptiven Statistik wurden die kontinuierlichen Variablen als Mittelwerte \pm Standardabweichung angegeben. Als Maß für die Veranschaulichung bei nicht normalverteilten Merkmalen wurde der Medianwert (50. Perzentil) inklusive Angabe der Spannweite benutzt. Eventuelle Mittelwertunterschiede zweier abhängiger Gruppen (Assessment-Ergebnis vor GFK vs. Assessment-Ergebnis nach GFK) wurden beim Vergleich von Merkmalen mit Normalverteilung mittels gepaarten T-Test ermittelt, bei nicht normalverteilten Messgrößen wurde der Wilcoxon-Test für Paardifferenzen herangezogen. Grundsätzlich wurde das Signifikanzniveau eines Ergebnisses bei einem $p<0,05$ als signifikant bewertet. 


\section{Ergebnisse}

Zur Auswertung gelangten die Daten von insgesamt 58 Patienten (21 männliche Patienten, 37 weibliche Patienten) mit akut exazerbierter COPD. Das mittlere Alter betrug 74,8 $\pm 6,8$ Jahre. Der Body Mass Index (BMI) lag im Mittel bei $26,2 \pm 6,6 \mathrm{~kg} / \mathrm{m}^{2}$. Die Lungenfunktionsprüfung wurde im Median am 6.Tag des stationären Aufenthaltes durchgeführt (4.-21.Tag). Das in einer Sekunde maximal exspirierte Volumen $\left(\mathrm{FEV}_{1}\right)$ lag für das gesamte Kollektiv $(n=58)$ bei durchschnittlich $0,82 \pm 0,341$ bzw. $38 \pm 13 \%$ des individuellen Sollwertes. Diese lungenfunktionsanalytischen Werte verdeutlichen die klinische Relevanz der Atemwegsflusslimitation innerhalb des untersuchten Kollektivs. Gemäß der COPD-Schweregradeinteilung nach der aktuellen GOLD-Klassifikation von 2010 [22] befanden sich 19\% der Patienten im Stadium II, 20,7\% im Stadium III und 60,3\% im Stadium IV. Im Durchschnitt waren die weiblichen Patienten älter, die männlichen Patienten hatten dagegen eine höhere Komorbidität. Die deskriptiven Daten des Patientenkollektivs finden sich in $\bullet$ Tab. 1.

Die frührehabilitative Komplexbehandlung wurde im Median am 3. Tag (1.-22. Tag) des stationären Aufenthaltes in der pneumologischen Akutklinik begonnen. Die mediane Verweildauer in der Akutklinik lag bei 19 Tagen (14-42 Tage). Die Dauer der GFK betrug im Median 17 Tage (9-29 Tage) und beinhaltete median 26 Behandlungseinheiten (20-57 Einheiten). Das multiprofessionelle Rehabilitationsteam konnte insbesondere in den Bereichen Mobilität (Timed-up-and-go, Median 19 [10-150] vs. 15 [7-120] Sekunden, $p<0,0001)$ und Selbsthilfefähigkeit (Barthel-Index, median 73 [5-95] vs. 95 [45-100], $p<0,0001)$ eine signifikante Verbesserung erreichen. Die Ergebnisse der Assessments bei Aufnahme und Entlassung sind in Tab. 2 aufgeführt und in den Bereichen Selbsthilfefähigkeit und Mobilität gegenüber gestellt. Grafische Darstellungen der Assessment-Verläufe für diese beiden Bereiche finden sich in den $\triangle \mathbf{A b b} .1$ und - Abb. 2.

Mit Hilfe des Sozialdienstes und der sozialen Assessments wurde bei 21 Patienten (36\%) erstmals der Antrag auf eine Pflegestufe gestellt, bei 4 Patienten (7\%) eine Höherstufung der Pflegestufe befürwortet und bei 9 Patienten (16\%) eine AHB beantragt. Bei 28 Patienten (48\%) wurden Hilfsmittel (z.B. Rollatoren, Sauerstoffgeräte, Pflegebetten) verordnet und zusammen mit anderen wohnumfeldverbessernden Maßnahmen für eine optimierte Versorgung im ambulanten Bereich gesorgt.

\section{Diskussion}

Im Rahmen der multiprofessionellen Behandlung von COPD-Patienten erwies sich die Zusammenarbeit der beiden Teilgebiete Pneumologie und Geriatrie innerhalb des akut-pneumologischen Settings als außordentlich fruchtbar. Sowohl die inhaltliche als auch formale Realisierung der Vorgaben des OPS-Kodes 8-550 waren in vollem Umfang und bei jedem Patienten gewährleistet. Die Evaluationsmethoden leiteten sich dabei ausschließlich aus der Geriatrie $a b$, da es sich führend um eine Akutbehandlung handelte. Klassische Verlaufsparameter der Rehabilitationsmedizin in der Pneumologie (z.B. Evaluation der krankheitsspezifischen Lebensqualität, Erfassung des subjektiven Dyspnoeempfindens, Quantifizierung der körperlichen Leistungsfähigkeit etc.) kamen noch nicht zur Anwendung und sollten Gegenstand weiterer prospektiver Studien sein, die COPD-Patienten mit und ohne GFK vergleichen. Bei den beiden wesentlichsten Parame-
Tab. 1 Deskriptive Daten der Patientengruppe $(n=58)$. BMI= Body Mass Index. FEV1 = Forciertes exspiratorisches Volumen in 1 Sekunde.

\begin{tabular}{llll}
\hline Parameter & $\begin{array}{l}\text { Weibliche } \\
\text { Patienten } \\
\text { (n=37) }\end{array}$ & $\begin{array}{l}\text { Männliche } \\
\text { Patienten } \\
\text { (n=21) }\end{array}$ & $\begin{array}{l}\text { Gesamt } \\
\text { (n=58) }\end{array}$ \\
\hline $\begin{array}{l}\text { Alter } \\
\text { (Jahre) }\end{array}$ & $75,9 \pm 6,6$ & $72,8 \pm 7,0$ & $74,8 \pm 6,8$ \\
\hline $\begin{array}{l}\text { Größe } \\
\text { (cm) }\end{array}$ & $160,0 \pm 6,2$ & $174,3 \pm 5,2$ & $165,1 \pm 9,1$ \\
\hline $\begin{array}{l}\text { Gewicht } \\
\text { (kg) }\end{array}$ & $64,6 \pm 19,2$ & $82,2 \pm 15,0$ & $71,0 \pm 19,6$ \\
\hline $\begin{array}{l}\text { BMI } \\
\left.\text { (kg/m }{ }^{2}\right)\end{array}$ & $25,6 \pm 7,3$ & $27,1 \pm 5,2$ & $26,2 \pm 6,6$ \\
\hline $\begin{array}{l}\text { FEV1 } \\
\text { (I) }\end{array}$ & $0,70 \pm 0,21$ & $1,05 \pm 0,42$ & $0,82 \pm 0,34$ \\
\hline $\begin{array}{l}\text { FEV1 } \\
\text { (\% Soll) }\end{array}$ & $39,3 \pm 12,7$ & $36,2 \pm 13,3$ & $38,2 \pm 12,9$ \\
\hline $\begin{array}{l}\text { COPD-Stadium } \\
\text { (GOLD II/III/IV) }\end{array}$ & $(7 / 9 / 21)$ & $(4 / 3 / 14)$ & $(11 / 12 / 35)$ \\
\hline $\begin{array}{l}\text { Nebendiagnosen } \\
\text { (n) }\end{array}$ & $8,2 \pm 3,1$ & $9,5 \pm 3,2$ & $8,6 \pm 3,2$ \\
\hline
\end{tabular}

Tab. 2 Ergebnisse der geriatrischen Aufnahme- und Entlassungs-Assessments.

\begin{tabular}{|c|c|c|c|}
\hline Bereiche & $\begin{array}{l}\text { Aufnahme- } \\
\text { Assessment }\end{array}$ & $\begin{array}{l}\text { Entlassungs- } \\
\text { Assessment }\end{array}$ & $p$ \\
\hline \multicolumn{4}{|c|}{ Selbsthilfefähigkeit } \\
\hline $\begin{array}{l}\text { Barthel-Index } \\
\text { (Punkte) }\end{array}$ & $72,5(5-95)$ & $95(45-100)$ & $<0,0001$ \\
\hline \multicolumn{4}{|l|}{ Mobilität } \\
\hline $\begin{array}{l}\text { TUG } \\
\text { (Sekunden) }\end{array}$ & $19(10-150)$ & $15(7-120)$ & $<0,0001$ \\
\hline $\begin{array}{l}\text { TUG } \\
\text { (Schweregrad) }\end{array}$ & $3(1-5)$ & $2(1-5)$ & $<0,0001$ \\
\hline \multicolumn{4}{|l|}{ Kognition } \\
\hline $\begin{array}{l}\text { MMST } \\
\text { (Punkte) }\end{array}$ & $27(5-30)$ & - & - \\
\hline $\begin{array}{l}\text { MMST } \\
\text { (leichtgradige } \\
\text { Demenz) }\end{array}$ & $(8 / 58=13,8 \%)$ & - & - \\
\hline $\begin{array}{l}\text { MMST } \\
\text { (Demenz) }\end{array}$ & $(2 / 58=3,4 \%)$ & - & - \\
\hline \multicolumn{4}{|l|}{ Emotion } \\
\hline $\begin{array}{l}\text { GDS } \\
\text { ( } n \geq 1 \text { Punkt) }\end{array}$ & $(6 / 58=10,3 \%)$ & - & - \\
\hline
\end{tabular}

TUG = Timed-up-and-go: Schweregrad $1=<10$ Sek. (Alltagsmobilität uneingeschränkt), Schweregrad 2=11 - 19 Sek. (geringe Mobilitätseinschränkung), Schweregrad 3 $=20-29$ Sek. (funktionell relevante Mobilitätseinschränkung), Schweregrad $4=$ $>30$ Sek. (ausgeprägte Mobilitätseinschränkung), Schweregrad $5=$ Bettlägerigkeit (Immobilität); MMST = Mini-Mental-Status nach Folstein: Schweregrad 1=24-30 Punkte (normal), Schweregrad 2 = 17 - 23 Punkte (leichtgradige Demenz), Schweregrad 3 (Demenz); GDS = Geriatrische Depressionsskala (Short Version GDS-4).

tern (BI + TUG) des geriatrischen Assessments konnte unter Einbeziehung der GFK eine signifikante Verbesserung erreicht werden. Die GFK innerhalb einer akut-pneumologischen Behandlung kann als gut durchführbar angesehen werden.

Die Ergebnisse dieser Arbeit lassen die Vorteile der frührehabilitativen Komplexbehandlung für geriatrische Patienten in der pneumologischen Akutbehandlung bereits erkennen und stellen die Grundlage für weitere Untersuchungen dar. Vergleichende Studien sollten künftig zeigen, ob - ebenso wie bei der Behandlung des Schlaganfalls - eine sehr früh einsetzende Reha-Be- 


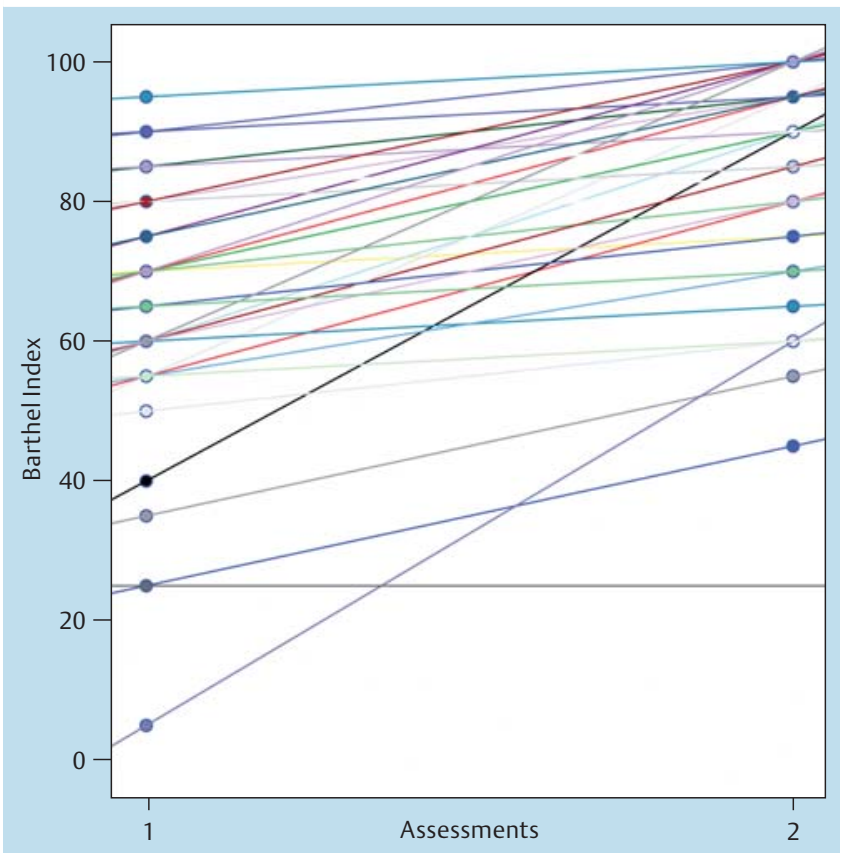

Abb.1 Ergebnisse des Aufnahme-Assessments [1] und Entlassungs-Assessments [2] für den Bereich Selbsthilfefähigkeit [Barthel-Index].

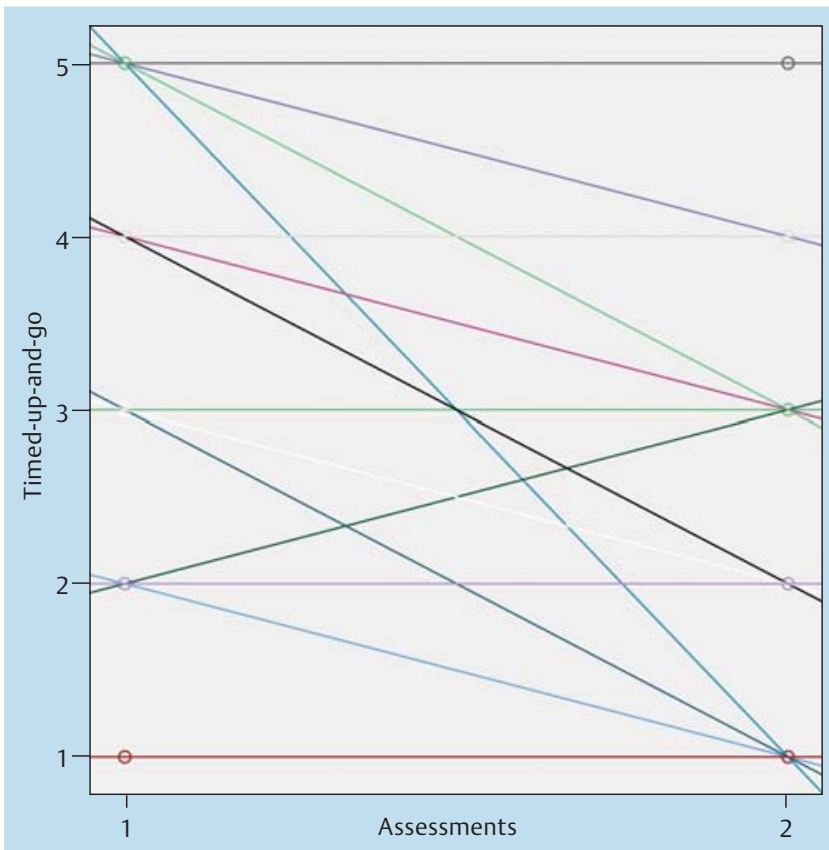

Abb.2 Ergebnisse des Aufnahme-Assessments [1] und Entlassungs-Assessments [2] für den Bereich Mobilität [Timed-up-and-go: Schweregrad 1 $=<10$ Sek. (Alltagsmobilität uneingeschränkt), Schweregrad 2 $=11-19$ Sek. (geringe Mobilitätseinschränkung), Schweregrad 3=20-29 Sek. (funktionell relevante Mobilitätseinschränkung), Schweregrad $4=>30$ Sek. (ausgeprägte Mobilitätseinschränkung), Schweregrad 5 = Bettlägerigkeit (Immobilität)].

handlung als integrativer Bestandteil der Akutbehandlung geriatrischer Patienten einer klassischen Reha, die in der Regel erst nach der pneumologischen Akutbehandlung einsetzt, überlegen ist. Wesentliche Studienendpunkte solcher Untersuchungen sollten jene Parameter sein, die für die Langzeitbehandlung und klassische Reha-Maßnahmen bei COPD-Patienten aussagekräftig sind. Dazu gehören Exazerbationsfrequenz, Hospitalisierungsrate und -dauer, Lebensqualität und Luftnotempfinden. Weiterhin untersucht werden müssen dabei der Nutzen geriatrischer Reha-Indikatoren und deren Korrelation zu pneumologischen Reha-Indikatoren.

Die GFK in der akutstationären Pneumologie im Rahmen einer Leistungserbringung gemäß Paragraph 108 SGB V als völlig neue Form der pneumologischen Rehabilitation tritt nicht in Konkurrenz mit bereits vorhandenen Strukturen (klassische stationäre Rehabilitation gemäß Paragraph 111 SGB V oder ambulante pneumologische Rehabilitation in dafür spezialisierten Einrichtungen), sondern ist als zusätzliches Glied einer Rehabilitationskette zur Reduktion von Funktionsverlusten und Verbesserung der Prognose zu verstehen.

\section{Unterstützung}

Diese Studie wurde unterstützt durch die Stiftung Oskar HeleneHeim, Berlin.

\section{Interessenkonflikt}

Die Autoren geben an, dass kein Interessenkonflikt besteht.

\section{Literatur}

1 Nici L, Donner C, Wouters E et al. American thoracic society/european respiratory society statement on pulmonary rehabilitation. Am J Respir Crit Care Med 2006; 173: 1390-1413

2 Puhan MA, Scharplatz M, Troosters $T$ et al. Respiratory rehabilitation after acute exacerbation of COPD may reduce risk for readmission and mortality a systematic review. Respir Res 2005; 6: 54

3 Puhan M, Scharplatz $M$, Troosters T et al. Pulmonary rehabilitation following exacerbations of chronic pulmonary disease. Cochrane Database Syst Rev 2009: 01 CD005305

4 Clini E, Ambrosino N. Rehabilitation in COPD patients: evergreen in pneumology and beyond. Eur Respir J 2011; 38: 514-515

5 Schultz K, Bergmann KC, Kenn K et al. Effektivität der pneumologischen Anschluss Rehabilitation (AHB): Ergebnisse einer multizentrischen prospektiven Beobachtungsstudie. Dtsch Med Wochenschr 2006; 131: $1793-1798$

6 Lacasse YL, Brosseau S, Milne S et al. Pulmonary rehabilitation for chronic obstructive pulmonary disease (Cochrane Review). In: The Cochrane Library. 04. Chichester, UK: John Wiley \& Sons; 2004

7 Lacasse Y, Wong E, Guyatt GH et al. Metaanalysis of respiratory rehabilitation in chronic obstructive pulmonary disease. Lancet 1996; 348 (9035): 1115-1119

8 Lacasse Y, Goldstein R, Lasserson TJ et al. Pulmonary rehabilitation for chronic obstructive pulmonary disease. Cochrane Database Syst Rev 2006: 04 CD003793

9 Salman GF, Mosier MC, Beasley BW et al. Rehabilitation for patients with chronic obstructive pulmonary disease: metaanalysis of randomized controlled trials. J Gen Intern Med 2003; 18: 213-221

10 Burtin C, Decramer M, Gosseling $R$ et al. Rehabilitation and acute exacerbations. Eur Respir J 2011; 38: 702 - 712

11 Puhan M, Spaar A, Frey $M$ et al. Early versus Late Pulmonary Rehabilitation in Chronic Obstructive Pulmonary Diesease Patients with Acute Exacerbations: A Randomized Trial. Respiration [Epub ahead of print] 16. 082011

12 Ko F, Dai D, Ngai J et al. Effect of early pulmonary rehabilitation on health care utilization and health status in patients hospitalized with acute exacerbations of COPD. Respirology 2011; 16: 617-624

13 Clini E, Crisafulli E, Costi S et al. Effects of early inpatient rehabilitation after acute exacerbation of COPD. Respiratory Medicine 2009; 103: $1526-1531$

14 Seymour J, Moore L, Jolley C et al. Outpatient pulmonary rehabilitation following acute exacerbations of COPD. Thorax 2010; 65: 423-428

15 Mannino MD. COPD: epidemiology, prevalence, morbidity and mortality, and disease heterogeneity. Chest 2002; 121: 121-126 
16 Cumming TB, Thrift AG, Collier JM et al. Very early mobilization after stroke fast-tracks return to walking: further results from the phase II AVERT randomized controlled trial. Stroke 2011; 42: 153-158

17 Matsui H, Hashimoto H, Horiguchi $\mathrm{H}$ et al. An exploration of the association between very early rehabilitation and outcome for the patients with acute ischaemic stroke in Japan: a nationwide retrospective cohort survey. BMC Health Serv Res 2010; 10: 213

18 Celli BR, MacNee W. ATS/ERS Task Force. Standards for the diagnosis and treatment of patients with COPD: a summary of the ATS/ERS position paper. Eur Respir J 2004; 23: 932 - 946

19 Vogelmeier C, Buhl RC, Criée CP et al. Leitlinie der Deutschen Atemwegsliga und der Deutschen Gesellschaft für Pneumologie und Beatmungsmedizin zur Diagnostik und Therapie von Patienten mit chronisch obstruktiver Bronchitis und Lungenemphysem (COPD). Pneumologie 2007; 61: e1 - e40
20 Quanjer PH, Tammeling GJ, Cotes JE et al. Lung volumes and forced ventilatory flows. Report Working Party Standardization of Lung Function Tests, European Community for Steel and Coal. Official Statement of the European Respiratory Society. Eur Respir J 1993; 6 (Suppl. 16): 5 40

21 Borchelt $M$, Kolb G, Lübke $N$ et al. Gemeinsame Arbeitsgruppe der Bundesarbeitsgemeinschaft der Klinisch-Geriatrischen Einrichtungen e.V., der Deutschen Gesellschaft für Geriatrie e.V. und der Deutschen Gesellschaft für Gerontologie und Geriatrie e.V. Abgrenzungskriterien der Geriatrie V1.3. Stand 2004. [Online] Verfügbar unter: http://www.geriatrie-drg.de/public/docs/Abgrenzungskriterien Geriatrie_V13_16-03-04.pdf

22 Global Initiative for Chronic Obstructive Lung Disease (GOLD). Update 2010. http://www.goldcopd.com 